\title{
Identifying the Forged Regions on the Images Using Shared Memory Model
}

\author{
J.Anitha ${ }^{1}$ \\ ${ }^{1}$ Department of Computer Science \& Engineering, Vignan's Institute of Information \\ Technology (A), Visakhapatnam, AP, India, \\ ${ }^{1}$ anithanv28@gmail.com
}

\begin{abstract}
In this paper, the representation of some of the regions on the image which were using without the information of the actual image users was given. The images which were using on now days are with good quality and the forging of these images is a little bit difficult rather than the old images. Most of the existing systems are struggling to identify the same for the images. Hence in the current paper, a model known as copy move forge detection using the shared memory model is given thought and implemented. The primary job of us is to identify the number of region son the images were being copied and to inform the developers about the number of regions on the images forge and using for other purposes. Several image formats like the JPEG, PNG are given thought for checking the current model. As per result, it is observed that the current model is working excellent and the results shows that the model is well suited for identifying the various numbers of forged regions on the images.
\end{abstract}

Keywords: Image Processing, Genetic Algorithm, Clustering, Image Identification.

\section{Introduction}

Picture handling or computerized picture preparing were started working from the 1960s and organizations working on these tasks are Jet Propulsion Laboratory, MIT and Bell Labs[1][3]. Since the cost of processing in those days was too high, it was just until 1970s that the territory started to thrive with less expensive PCs and accessible devoted equipment. Pictures began to be prepared continuously for TV measures [2][4][5]. Presently, from 2000s with innovations of quick PCs and flag processors preparing had turned into the most widely recognized type of picture handling because of its adaptability and minimal effort. Picture processing is a strategy to upgrade crude pictures from cameras/sensors to put on satellites, space tests and flying machines.[6][7][8]

\subsection{IMAGE PROCESSING TECHNIQUES}

Various image processing techniques are,

IMAGE PORTRAYAL: Image characterization is considered as a capacity $f(x, y)$ where $x$ is the number of lines and $y$ is the number of segments of the picture. The crossing point of line and section is a pixel.

Article history:

Received (January 23, 2019), Review Result (March 11, 2019), Accepted (April 29, 2019) 
IMAGE PRE-HANDLING: Image pre-preparing strategy is utilized to set up the picture reasonable for the specific application. Picture pre-handling strategy incorporates scaling, extending, smoothing, and so on.

IMAGE UPGRADE: Image upgrading strategy is utilized to enhance the nature of the picture as pictures acquired from different sources like satellites and advanced cameras will contain a parcel of clamor. A portion of the improvement methods are Histogram alteration, Noise Filtering and so on.

IMAGE REPRODUCTION: Image recreation from articleions is a unique class of picture reclamation issues where a two-(or higher) dimensional protest is remade from a few onedimensional articleions.

IMAGE INFORMATION PRESSURE: Compression is an extremely fundamental device for filing picture information, the picture information exchange on the system and so on.

\subsection{INTRODUCTION TO FORGERY DETECTION}

Advanced pictures are difficult to control and alter because of accessibility of effective picture handling and altering programming. The location of malignant control with computerized pictures is forgery detection. Discovery strategy found can be ordered into two ways like dynamic technique and uninvolved strategy. A dynamic discovery technique works on the nearness of watermarking or the unique finger impression, which comprises of adding picture points of interest with a specific end goal as name, date, signature, and so on. While the latent technique comprises of recognizing imitations or copied questions in pictures without thinking about the data of the pictures. The correcting strategy which deals with controlling the computerized picture by changing its highlights without making detectable alterations of the substance of the picture. This can be either technical correcting or creative modifying.

\section{LITERATURE SURVEY}

Because of the sophisticate altering programming, computerized pictures can be effectively controlled and adjusted without leaving unmistakable pieces of information. It represents a genuine social issue concerning the amount of their substance can be trusted, regardless of whether it is true or altered, particularly as an observer in a court. Advanced picture legal sciences have risen to uncover computerized altering in pictures. There are a few sorts of altering, be that as it may, covering a few items from normal pictures is a typical type of advanced picture altering, known as duplicate move falsification. It is a particular kind of picture altering, where a piece of the picture is reordered on another piece of a similar picture. With a specific end goal to distinguish Copy-Move Forgery in a picture we have to comprehend the square based coordinating.

For the most part, a nature picture is probably not going to have two vast comparative districts. The errand of discovering Copy-Move imitation is to discover substantial comparative locales in a picture. Since the copied locales are obscure, on the off chance that we think about each conceivable pixel by the pixel, the computational many-sided quality will be exceptionally higher. Clearly, it is more down to earth to isolate the suspicious picture into hinders for recognizing the copied locales. So, the entire picture is separated into squares and discovery calculations are connected on those pieces to identify the altered zones.

Since the advanced pictures assume a huge part in improving the method for speaking to and exchanging thoughts adaptably, a consideration has been paid as of late towards exploring the 
reasonable instrument for breaking down and identifying falsification in the computerized pictures. This consideration was because of the most recent exercises in which a solitary protest inside the picture is copied a similar picture. Such exercises can be found in the duplicate move forge that considers a standout amongst the most known action goes for concealing the information. Normally, it is conceivable to distinguish the copied question by figuring and contrasting these premises and the entire picture.

\subsection{THE EXISTING SYSTEM}

The current system utilizes dynamic systems like watermarking or computerized marks to take care of the confirmation issue in pictures and to influence it to free from altering.

Issues with existing framework

The present forgery detection has following disadvantages,

The existing framework has constraints since they require human mediation or exceptionally prepared cameras like digital cameras with watermarking innovation in it.

This framework additionally requires the data of the first picture to recognize phony in a picture.

This framework depends totally on watermarks and unique picture data, yet by utilizing in fact progressed computerized photography instruments, one can alter, control or alter the pictures effortlessly without living evident visual hints, for which the framework neglects to distinguish in the greater part of the cases.

\subsection{PROPOSED SYSTEM}

Primarily the system wants details about original image to trace the amount of data on the image copied. But the copied data identification was done with the help of the copy move forge with shared memory model such that to identify the area or the region of the amount of data being copied from the original images. For the current images, from where the authors are trying to identify the amount of data copied and the amount of data being traced from the other source of images and other sources of data was required. Copy-Move forgery with shared memory model detection system can detect forgery of regions on images in digital images without any data about the original image and without any watermarks in the image.

\subsection{IMPROVED COPY-MOVE SMM FORGERY DETECTION}

The enhanced copy-move shared memory model forgery identification works in light of square coordinating method which is one of the uninvolved strategies of forge regions identification. In this, the calculation is actualized to recognize altering in computerized pictures. In this technique, at first the picture was taken as contribution for the framework and the picture is then changed over into shading palette. At that point, the entire picture is decayed into N BY N pieces. At that point, these pieces are requested by their pixel esteems. From those squares with little shading distinction are separated. Those are bunched in view of the convergence territory. At that point, shift through all the little bunches. Presently by checking the separation between the twin groups, we can recognize the duplicate move areas in the picture on the grounds that the altered picture will have same separation between any twins bunch focuses.

\section{METHODOLOGY}


The methodology that the authors considered in the current article was shown above. The images to be tested were given as input to the current system. Then, the images will be made into pieces or into tiny blocks of data. Then, these image pieces were made into groups by clustering based on the selection of the regions on the images. These model of pieces will be brought together by using the shared memory model such that to make them as set of images. Then these images will be compared with the other set of images for the matching identification and for the details of the images were discussed in the results section. The entire methodology followed in the current model was explained or given in the diagram model and it is shown as follows,

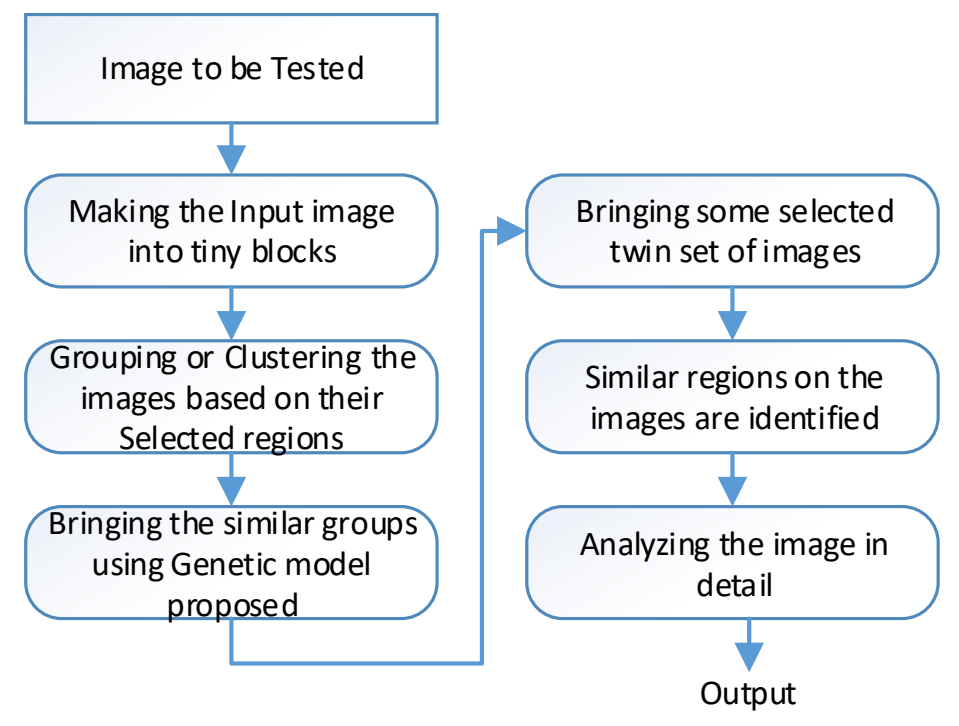

Figure.1 Methodology Adopted in the Model

\subsection{System Design}

The interfacing unit of the MATLAB as discusses in detail and the image model was shown in figure 2 . 


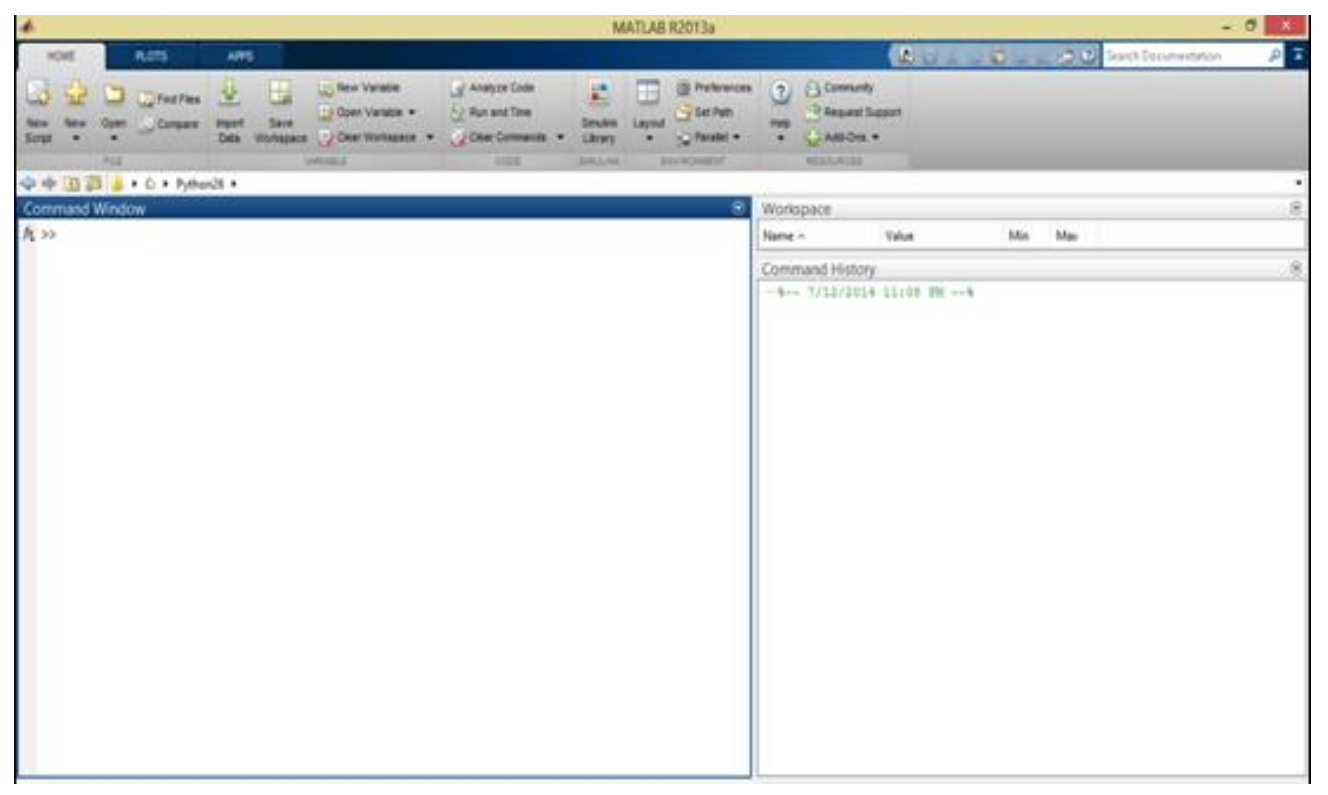

Figure. 2 MATLAB Interface

Designing Graphical User Interfaces

Utilizing GUIDE (Graphical User Interface Development Environment), you can format, plan, and alter custom graphical UIs. You can incorporate basic controls, for example, list boxes, pull-down menus, and push catches, and in addition MATLAB plots. Graphical UIs can likewise be made automatically utilizing MATLAB capacities.

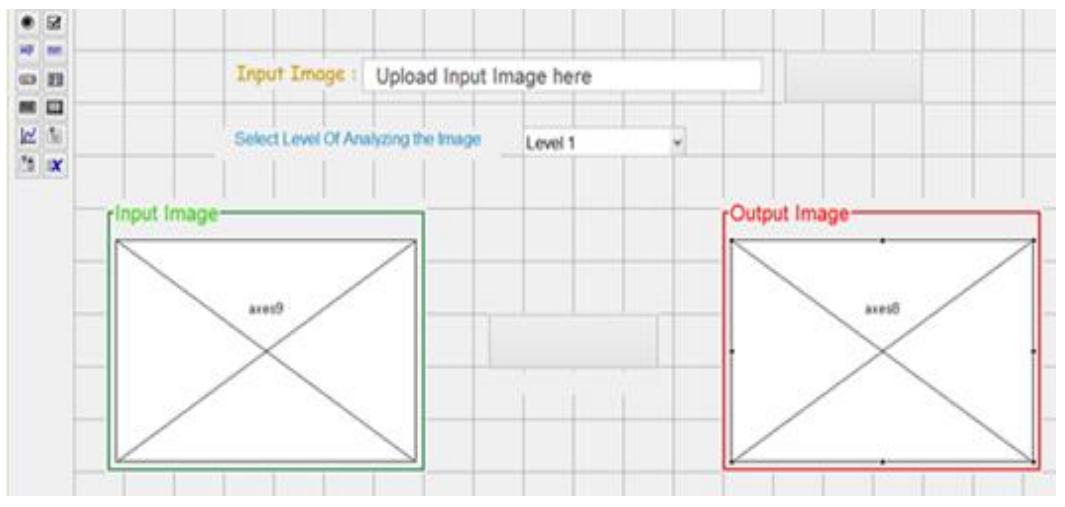

Figure. 3 GUI Interface

This screen enables the client to begin his/her communication with the current framework. This screen has a choice to transfer a picture which will be the information picture for the framework. In the wake of tapping on the transfer catch in the appreciated screen a discourse box will show up where the client needs to peruse and select the information picture. In the exchange box the client needs to choose "All Files" in kind of records menu and peruse for the information document by heading off to the coveted index and select it and tap on open catch in the discourse box. 


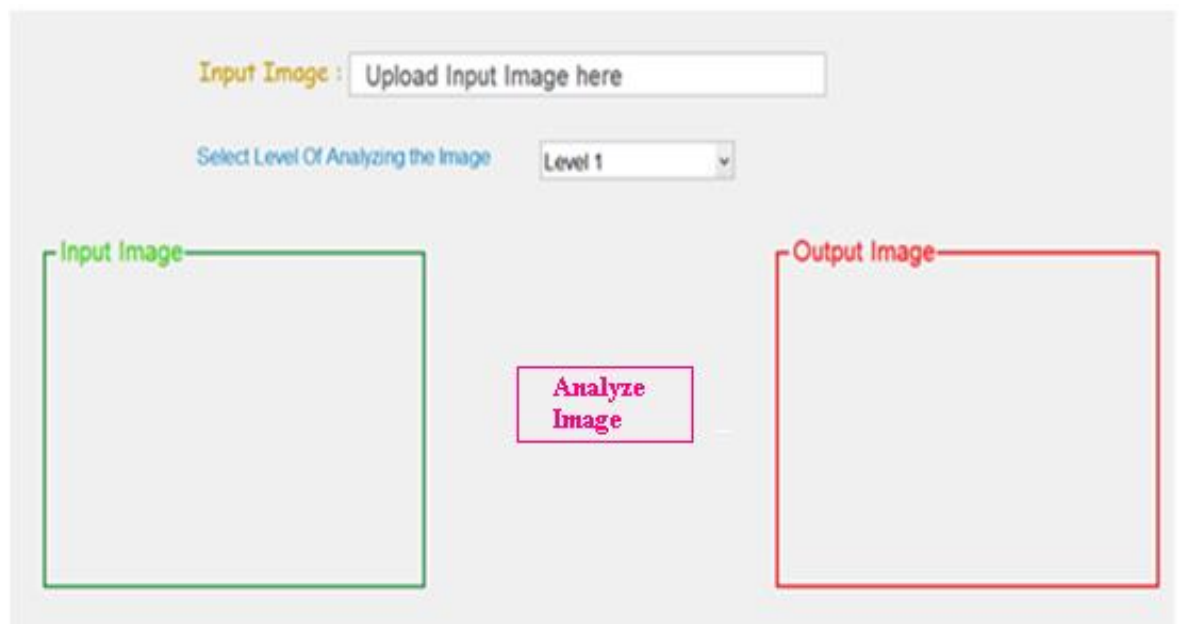

Figure. 4 After Design

Subsequent to choosing the information picture the client can see this screen which demonstrates the way of the information picture, shows the picture under Input Image, and can pick the level up to which the framework needs to break down the picture for identifying the altering.

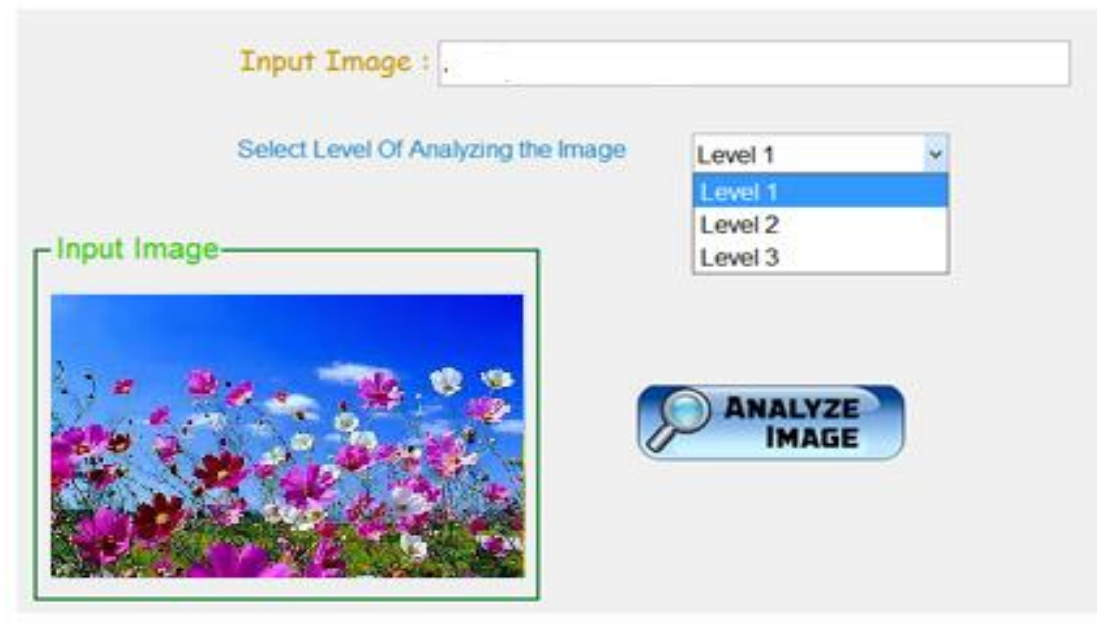

Figure. 5 After Design

At the point when the client taps on break down picture catch the transfer picture catch consequently gets impaired and checking the picture for location of duplicate moved areas in the info picture begins. A message is shown on this screen saying "Investigating picture please wait..." 


\section{Input Image : es/pythonfimagel pme}

Select Level Of Analyzing the Image

Level 1

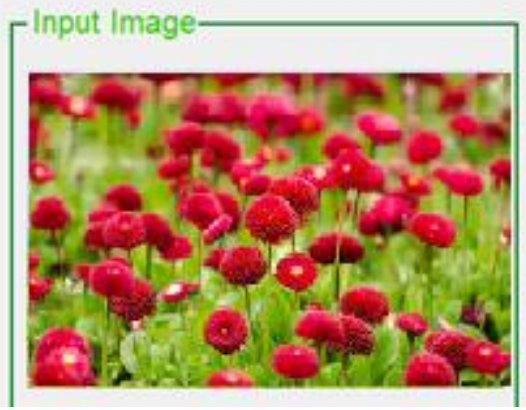

\section{Analyze \\ Image}

Figure. 6 After Design

Once if examining of the information picture is done a yield picture is shown under a yield picture mark, which demonstrates the distinguished duplicate move areas in the given picture. This is the result screen and if the client needs to investigate the picture again he/she can change the Level of dissecting and can examine the picture again or can basically transfer a picture again utilizing the transfer catch.

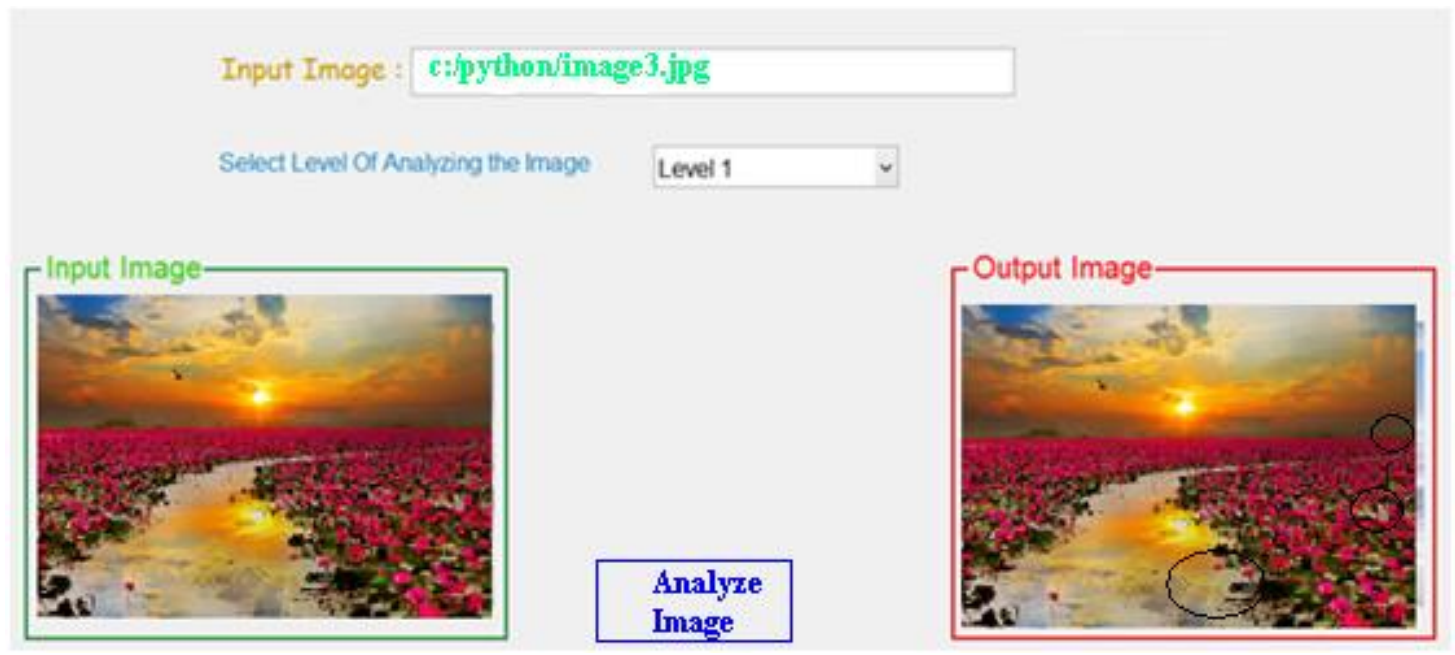

Figure. 7 After Design and Analyze 


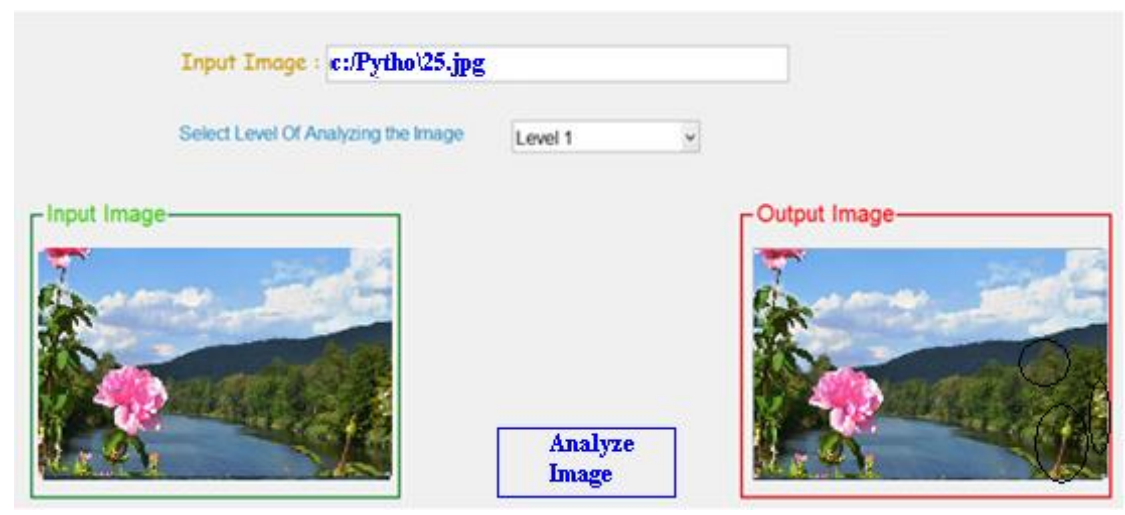

Figure. 8 Output after Design

\section{CONCLUSIONS}

In the current paper, the detection of forged region son the images was discussed in detail. By using the current application, users can find the forged regions easily on images having good quality too. This model helps to solve this problem easily. The images should not contain any water mark, and then the expected results from the current article were at a good rate of working. The current model of incorporating shared memory model had given the better results as per expectation. These stages were successively executed since the aftereffect of the past stage is utilized as a part of the following stages. The proposed strategy depends on the square coordinating idea where the picture is isolated into pieces.

\section{References}

[1] Sukhada Aloni "Content Based Image Retrieval in Biomedical Images Using SVM Classification with Relevance Feedback", International Journal of Scientific and Research Publications, Vol.3, No.11, pp.1-7, November (2013).

[2] M. Eitz, K. Hildebrand, T. Boubekeur, and M. Alexa, "An evaluation of descriptors for large-scale image retrieval from sketched feature lines" Computers \& Graphics, Vol.34, pp.482-498, (2010). DOI: 10.1016/j.cag.2010.07.002

[3] S. Belongie, J. Malik, and J. Puzicha. "Shape matching and object recognition using shape context". PAMI Vol. 24, No.4, pp.509-522, (2002). DOI: 10.1109/34.993558

[4] X. Fu, Y.Li, R. Harrison, and S. Belkasim, (2006) "Content based image retrieval using Gabor-zernike features.18th international conference on pattern recognition IEEE.DOI: 10.1109/ICPR.2006.408

[5] M. M. Rahman, P. Bhattacharya, and B. C. Desai, "A framework for medical image retrieval using machine learning and statistical similarity matching techniques with relevance feedback," IEEE Trans. Inf. Tech. Biomed., Vol. 11, No. 1, pp. 59-69, Jan. (2007).DOI: 10.1109/titb.2006.884364

[6] T. M. Lehmann, B. B. Wein, J. Dahmen, J. Bredno, F. Vogelsang, and M. Kohnen, "Content-based image retrieval in medical applications, A novel multi-step approach,” Proc. SPIE, Vol. 3972, pp. 312-320, (2000).DOI: 10.1267/METH04040354

[7] H. Muller, A. Rosset, J. Vallee, and A. Geissbuhler, "Integrating contentbased visual access methods into a medical case database," in Proc. Med Inf. Eur., St Malo, France, pp. 480-485.

[8] H. Muller, J. Kalpathy Cramer, I. Eggel, S. Bedrick, S. Radhouani, B. Bakke, C. E. Kahn, Jr., and W. Hersh, "Overview of the CLEF 2009 Medical Image Retrieval Track," 10th Workshop of the Cross-Language Evaluation Forum, CLEF 2009, Corfu, Greece, Sep. 30-Oct. 2, 2009, Proceedings of LNCS, Vol. 6242, pp. 7284, (2010).DOI: 10.1007/978-3-642-15751-6_8 